\title{
A VORONOI DiAgRAM-BASED APPROACH FOR ANALYZING AREA COVERAGE OF VARIOUS NODE DEPLOYMENT SCHEMES IN WSNS
}

\author{
G Sanjiv Rao ${ }^{1}$ and V Vallikumari ${ }^{2}$ \\ ${ }^{1}$ Associate Professor, Dept of CSE, Sri Sai Aditya Institute of Science \\ And Technology, Surampalem, AP India. \\ ${ }^{2}$ Professor, Dept of CS\&SE, College of Engineering, Andhra University \\ Visakhapatnam, AP, India.
}

\begin{abstract}
With recent advances in electronics and wireless communication technologies, the Wireless Sensor Networks (WSNs) are being used in wide range of applications of military, ecological, health related areas. Due to their resource constraints such as, sensor node's size, memory, and processing capabilities, the scale of deployment of WSNs requires careful decisions with respect to various performance measures. In the last few years, there has been a tremendous interest on development of large-scale wireless sensor networks as a basic issue to be addressed as it can influence the performance metrics of WSNs such as, coverage, connectivity, resilience and scalability requirements. Many deployment schemes have been proposed for wireless sensor networks. In this paper, we survey six deployment models random, rectangular-grid, square-grid, triangular-grid, hexagonal-grid, and hybrid deployments schemes and analyze their implications on network area coverage in WSNs. More generally, under some deployment assumptions on an irregular geographical map for a defense monitoring, we show the analytical and simulation-based results of a WSN made up of mica2 motes using the deployment knowledge to motivate the use of these emerging paradigms in order to achieve higher network area coverage. To apply deployment schemes on irregular geographical target area, we propose to include the concept of voronoi diagram based approach in WSNs, to provide a way of dividing an irregular geographical area into a number of regular regions. We have been configured the sensor node parameters such as sensing, temperature, energy capabilities using mannasim based on NS-2.34.
\end{abstract}

\section{KEYWORDS}

Wireless Sensor Network, Deployment Knowledge, Coverage.

\section{INTRODUCTION}

A wireless sensor network (WSN) is a collection of small, low cost and battery powered devices called sensor nodes. The sensor nodes are also called as MICA2-DOT motes [3]. A WSN is composed of densely deployed sensor nodes in a geographical area with a purpose of collecting and processing some useful data, and transmitting the data to nearby base stations for further processing. The transmission between the sensors is done by short range radio communications. The base station is assumed to be computationally well-equipped whereas the sensor nodes are resource- starved. A hierarchical wireless sensor network is explained in [1].

Usually the sensor networks are composed of one or more base stations and many sensor nodes scattered in a sensor field (i.e., deployment field or target field) as shown in Figure. 1each of these scattered sensor nodes has the capability to collect data and route data back to the base station. A base station or sink node serves as a gateway to another network, which is a powerful data processing unit, and also responsible for network management, collecting and performing costly operations on behalf of sensor nodes. As explained in a broadcast authentication scheme in WSNs [2], a base station acts like a commander, and broadcasts the commands to the sensors, and

DOI : 10.5121/ijasuc.2014.5202 
upon receiving those commands, sensors send results back to the base station. To route data back to the base station, sensor nodes use a broadcast or multi-hop communication paradigm instead of point-to-point communications. The base station may communicate with the task manager node via Internet or Satellite.

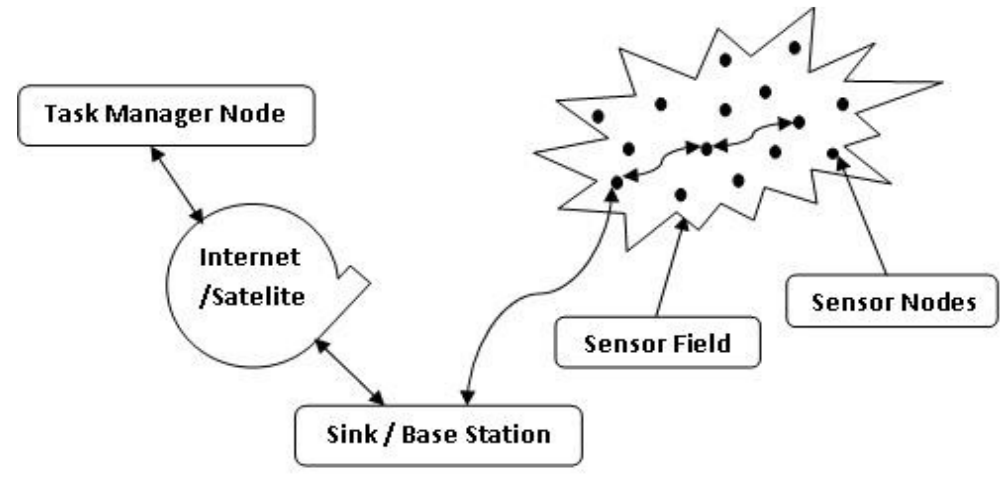

Figure 1. Sensor Nodes scattered in a traget field

Using different sensors, WSNs can be implemented in many application areas including, security, entertainment, health care monitoring, automation, industrial monitoring, public utilities, and asset management. In fact, the sensor networks need to have some unique features and application specific requirements, which made them different from ad hoc networks as listed in [12].

For many applications, sensors networks are usually deployed in sensing area to collect some information, and exhibits frequent changes in its topology. Moreover, WSN devices have severe resource constraints in terms of energy, computation, memory and require large number of nodes. Therefore, deploying sensor nodes to provide complete area coverage is a basic issue to be addressed. Eight alternative deployment schemes have been proposed in [6]. Depending upon the application requirements, node deployment can be either, application specific deterministic deployment, or random deployment or grid based deployment. Deterministic deployment is suitable for small-scale applications, where the sensors are placed directly in the required region. For large-scale applications, random deployment is suitable, where sensors are thrown randomly to form a network. Grid-based deployment is suitable for moderate to large-scale applications, where individual sensors are placed exactly at grid points. In this paper, we make a comparison on the amount of network coverage for various sensor node deployment strategies namely, random, square-grid, rectangular-grid, triangular-grid, hexagonal-grid, and hybrid deployment schemes.

To demonstrate our approach more analytically, we consider an irregular shaped real geographical map of Sri Lanka country as a target area for the wireless sensor network deployment with few human soldiers are monitoring the area within their eye vision at fixed check-points. We also propose to make use of a voronoi diagram based approach to divide the irregular target area into regular regions. We have simulated the node deployment strategies using the simulation tools (such as Network Simulator-2.34 [11] and Mannasim [13]).

The rest of this paper is organized as follows: Section 2 provides preliminary notions that are needed throughout the paper. Section 3 presents simulation based analysis of various node deployment schemes. Section 4 focuses on comparative analysis of the deployment schemes with respect to network coverage by considering an irregular shape geographical map as a target area and Section 5 concludes our work.

\section{Preliminaries}

Our analysis will be based on some key assumptions made from the concept of voronoi diagram based approach in WSNs, to provide a way of dividing an irregular geographical area into a number of regular regions, for the WSN deployment, and calculating the area coverage for 
International Journal of Ad hoc, Sensor \& Ubiquitous Computing (IJASUC) Vol.5, No.2, April 2014

various geometric shapes inscribed in a two-dimensional irregular geographical map for defense monitoring.

\subsection{Assumptions and Notations Used}

- For instance, consider a geographical area map of Sri Lanka country for defense monitoring (i.e. target area for the WSN deployment), be in an irregular shape, and, with different highway checkpoints and landmarks etc.

- Assume, each of the human soldiers are fixed at some static and plane check points and monitoring the area which is under their eye vision.

- After being divided, each regular geometric shape within the irregular target area be approximated with straight line edges and the real geographical distance is taken from Google earth application [16].

\section{The following are some notations used throughout this paper.}

- Let $T_{r}$ be the irregular shape defense monitoring target region, for WSN deployment.

- Let $\mathrm{A}$ be the area of the target region $T_{r}$

- Let each of the human soldiers are located and monitoring the target area within their eye vision at some fixed and plane check points, such as, $v_{1}, v_{2}, v_{3}, v_{4}, \ldots \ldots \ldots \ldots v_{n}$.

- Let $X_{m}$ be the amount of contribution of each soldier.

- Let $r$ be the maximum distance covered through a human soldier eye vision in a single direction while monitoring the target area.

- Let $\pi r^{2}$ be the area of target region under human soldier eye vision in all directions (i.e. in a circular shape).

- Let $G(x)$ is the overlapping eye vision area of two or more human soldiers.

- Let $c_{1}, c_{2}, c_{3}, c_{4}, \ldots \ldots c_{n}$ (where $\mathrm{n}$ is a fixed value) are the boundary check points of the target area $T_{r}$.

- Let $S_{1}, S_{2}, S_{3}, S_{4}, \ldots S_{n}$ are the number of resolved regular geometric shapes approximated with straight edges, within the irregular target region $T_{r}$.

- Let $R_{1}, R_{2}, R_{3}, R_{4}, \ldots \ldots R_{n}$ are the areas of resolved regular shapes within the target region $T_{r}$.

- Let $u_{1}, u_{2}, u_{3}, u_{4}, \ldots \ldots \ldots \ldots u_{n} \mathrm{u} 1, \mathrm{u} 2 \ldots . . \mathrm{un}$ are the uncovered areas in the target field after being divided into number of regular geometric shapes.

- If we consider a largest square inside the target region, then, the maximum coverage area of the contained square is given by,

$$
\text { Area of Square }=\text { Side } * \text { Side }
$$

- If we consider a largest rectangle inside the target region, then, the maximum coverage area of the contained rectangle is given by,

$$
\text { Area of Rectangle }=\text { width } * \text { height }
$$

- For instance, if we have an triangle inscribed in the target region, then, let a, b, c be the lengths of the sides of a triangle. The area is given by,

$$
\text { Area of Triangle }=\sqrt{p(p-a)(p-b)(p-c)}
$$

where $\mathrm{p}$ is half the perimeter, or $(a+b+c) / 2$.

- Let us consider a hexagon is inscribed in the target region, assuming it's a regular hexagon, then

$$
\text { Area of a Hexagonal }=\frac{S^{2} N}{4 \tan \left(\frac{180}{N}\right)}
$$

Where $\mathrm{S}$ in the length of any side, $\mathrm{N}$ is the number of sides, tan is the tangent function calculated in degrees. 
International Journal of Ad hoc, Sensor \& Ubiquitous Computing (IJASUC) Vol.5, No.2, April 2014

- If we consider a hybrid deployment (i.e. Combination of all of the above geometric shapes) inside the target region, then,

$$
\text { Hybrid Deployment }=\text { Area }(S 1+S 2+S 3+S 4+S 5+S 6+S 7)
$$

\subsection{Simulation Tools Used}

- NS-2.34 is a discrete event network simulator that has been developed in 1989 as a variant of the REAL network simulator. Implementation and simulation of a network using NS-2 consists of 4 steps (1) Implementing the protocol by adding a combination of C++ and OTcl code to NS-2's source base (2) Describing the simulation in an OTcl script (3) Running the simulation and (4) Analyzing the generated trace files. NS-2 is a powerful tool for simulating ad-hoc networks. But to simulate WSNs in NS-2, we need to have an additional module namely mannasim to represent the protocols, that are specific to WSNs.

- MANNASIM is a framework for WSNs simulation, and is based on NS-2. It introduces new modules for design, development and analysis of different WSN applications. An excellent survey on simulation tools is given in [15]. WSN simulations with mannasim for various deployment schemes have been explained in [14].

\section{Deployment Schemes for WSNs}

In WSNs, the major challenge is the deployment of the nodes in the deployment region to satisfy continuous sensing with extended network lifetime while maintaining uniform coverage. In this section we focus on simulation and analysis of various node deployment strategies (such as, random, rectangular-grid, square-grid, triangular-grid, hexagonal-grid, and hybrid deployment schemes) that have been developed for wireless sensor networks as described in [6]. We analyze network coverage as a performance metric for each of these deployment schemes in the next section.

\subsection{Simulation of Random Deployment}

Random approach for node deployment is deeply discussed in [10]. In this deployment, as shown in below Figure. 2 we visualize a simulated sensor network with 20 nodes deployed with random deployment scheme, each of the sensors has equal probability of being placed at any point inside a given target area.

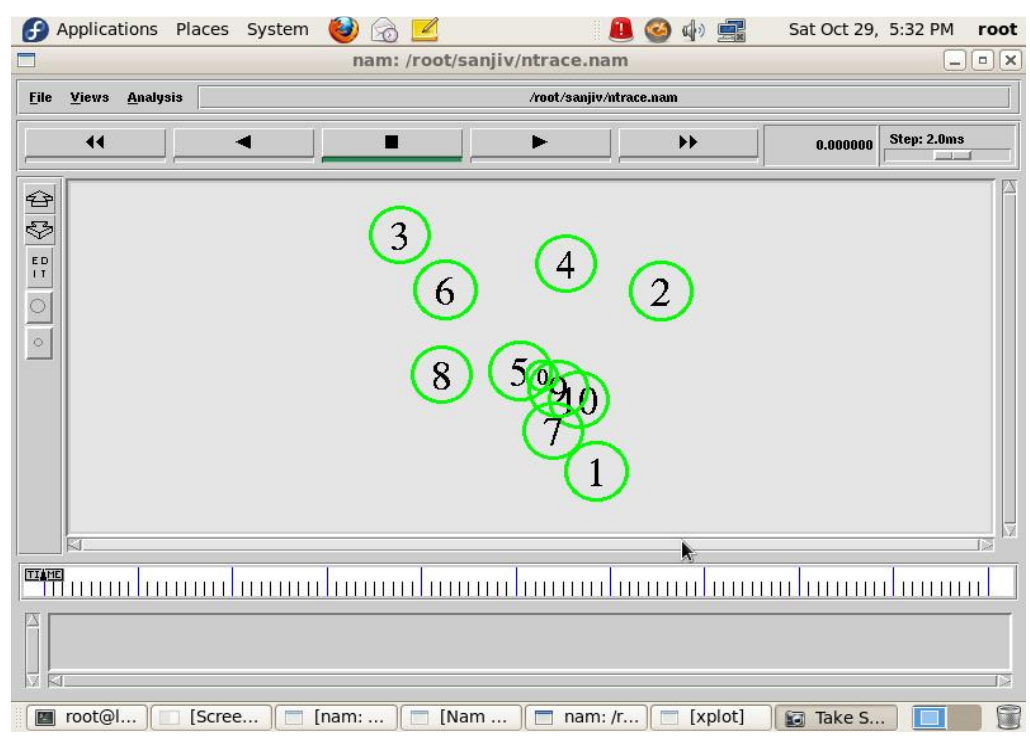

Figure 2. Simulation of Random Deployment 
International Journal of Ad hoc, Sensor \& Ubiquitous Computing (IJASUC) Vol.5, No.2, April 2014

\subsection{Simulation of Grid-Based Node Deployment Scheme}

In [4], it has state that grid deployment is an attractive approach for moderate to large-scale coverage-oriented deployment due to its simplicity and scalability. Based on these considerations, we focus on popular grid layouts such as square-grid, rectangular-grid, triangular-grid, and hexagonal-grid.

\subsubsection{Square Grid}

As described in [4], we investigate a square grid because of its natural placement strategy over a unit square. In the below Figure. 3 we present the simulation results of a square grid deployment of 9 sensor nodes.

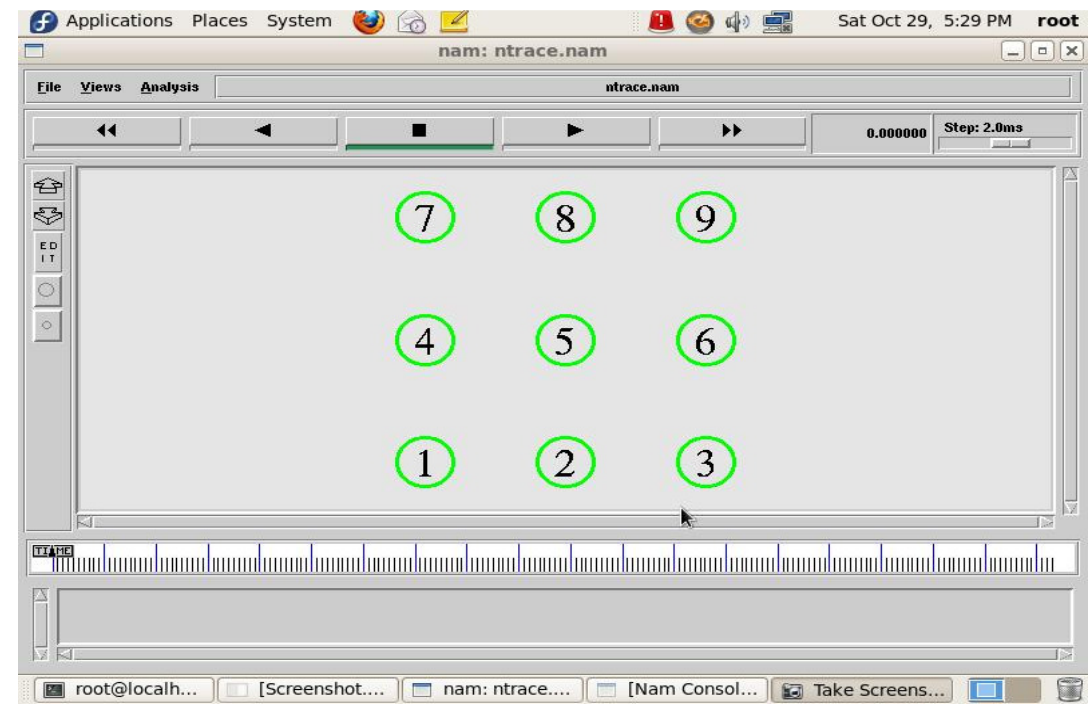

Figure 3. Simulation of Square Grid Deployment

\subsubsection{Rectangular Grid}

Square Grid: In below Figure. 4 shows the visualization of a simulated sensor network with 20 nodes deployed with rectangular grid deployment scheme. The desired distance between consecutive droppings is achieved by controlling the time intervals. However, due to placement errors, this ideal deployment is not realistic.

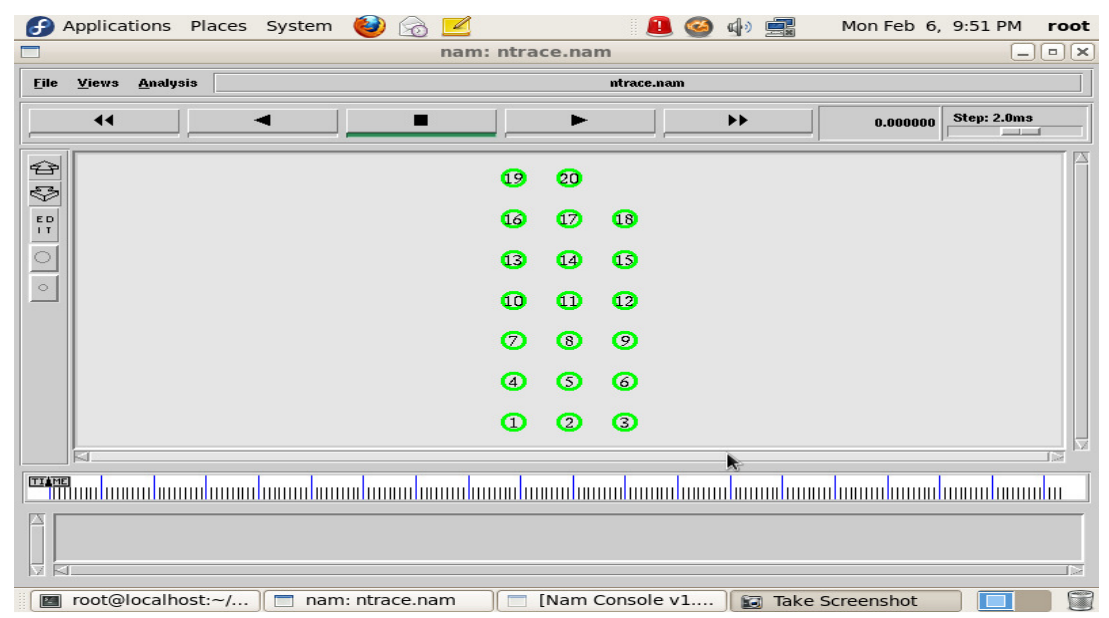

Figure 4. Simulation of Rectangular Grid Deployment 


\subsubsection{Triangular Grid}

As explained in [9], in this triangular deployment scheme can cover a sensing area fully. It divides the target area into cells. Each cell represents the triangular-shaped sensing area. In the below Figure. 5 we presented the simulation-based result of triangular deployment scheme. The below figure visualizes triangle deployment of 16 nodes among which node 0 represents sink node.

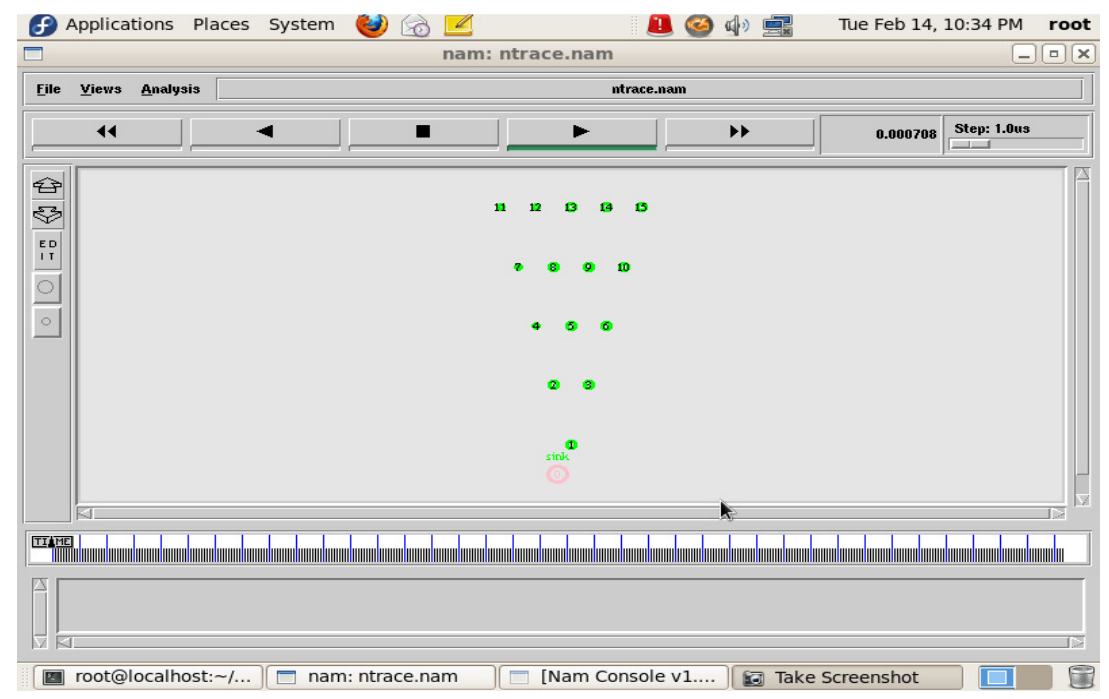

Figure 5. Simulation of Triangular Deployment

\subsubsection{Hexagonal Grid}

In this paper, one of the main goals of our work is to improve the covering area of interest. As explained in [6], we consider the simulation of a semi-regular tiling which uses triangle and hexagon in the two dimensional plane instead of considering a regular polygon which has the same side lengths and interior angle as described in [7]. As specified in [8], a tiling or tessellation of the plane is a process which uses a collection of polygons called tiles to cover the plane without gaps or overlaps. The below Figure. 6 shows simulation of 24 nodes using hexagonal grid deployment, where target field is partitioned into multiple hexagonal grids.

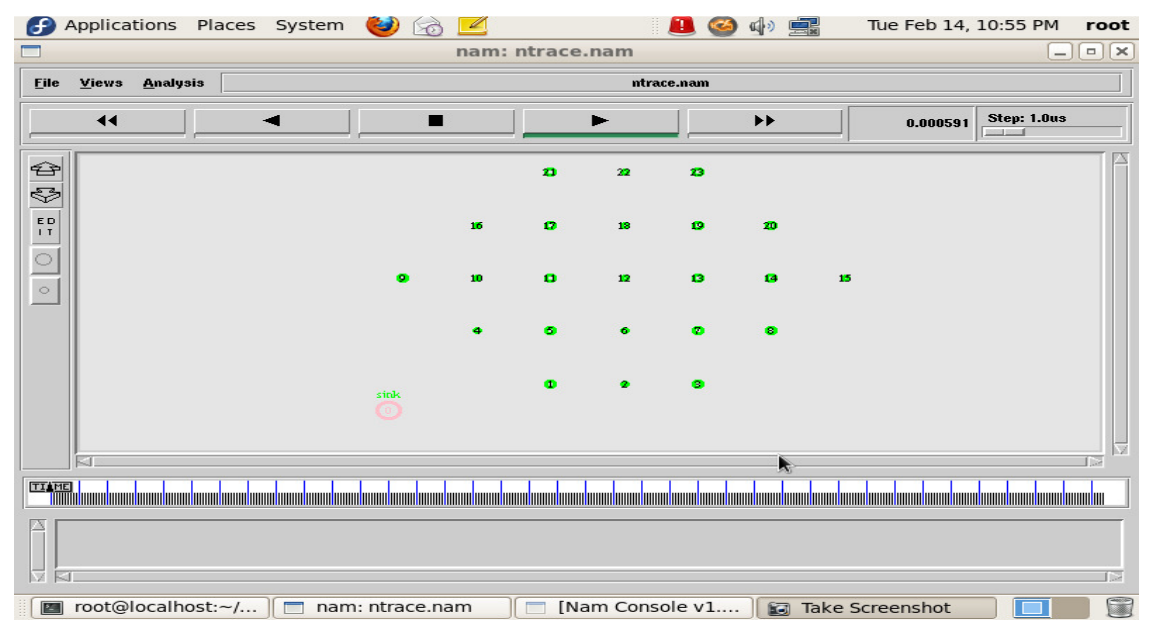

Figure 6. Simulation of Hexagonal Deployment 


\subsection{Hybrid Deployment}

As discussed in [6], in hybrid deployment, the target field consists of nodes deployed using various schemes such as random, rectangular-grid, square-grid, triangular -grid and hexagonalgrid deployments to improve network coverage.

Compared with existing schemes, hybrid node deployment scheme requires a shorter transmission range and achieves a higher coverage. In below Figure. 7 shows the simulation result of 44 nodes deployed in a hybrid deployment which is a combination of all other schemes specified in the previous sections. In this simulation nodes $0,11,22,33$, and 44 are the sink nodes.

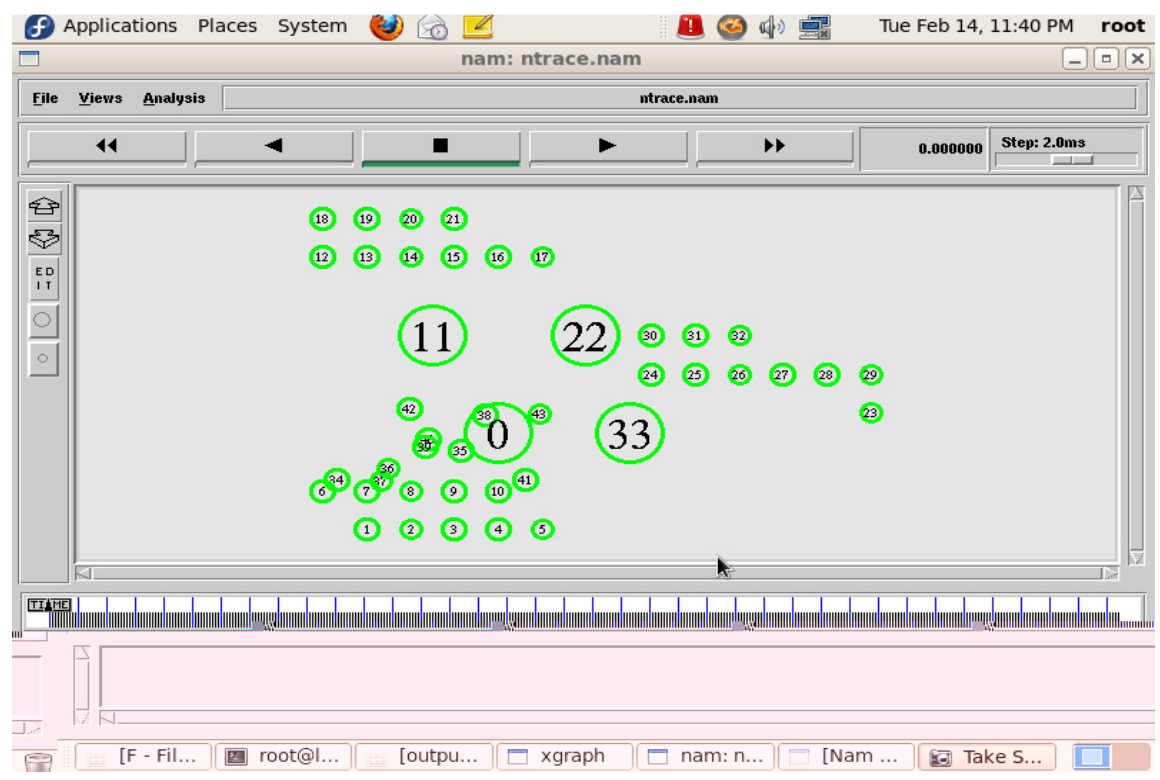

Figure 7. Simulation of Hybrid Deployment

\section{Related Work Done}

In this section, we present an analysis of various sensor node deployment schemes with respect to network coverage by considering different regular geometric shapes inside the real geographical map. Through this study we provide a comparative analysis of these deployment schemes with respect to network performance in terms of coverage.

\subsection{Analysis of Area Coverage}

For analyzing area coverage for various deployment schemes, we consider a real geographical map of Srilanka country, with an irregular shape, and, which has a total area of 65,610 km2, with $64,740 \mathrm{~km}^{2}$ of land and $870 \mathrm{~km}^{2}$ of water.

Let the Target Region $T_{r}=64,740 \mathrm{~km}^{2}$

We also consider different regular geometric shapes within the irregular geographical map, based on the voronoi diagram approach. In fact, to make our analysis easy, we approximated all of the geometric shapes with straight edges, and gather the real geographical distances between the edges of the geometric shapes in kilo meters from Google earth application [16]. 


\subsubsection{Analysis of Area Coverage for Random Deployment}

Analysis of Area Coverage for Random Deployment: As explained in [5], random deployment refers to the situation in which sensor nodes are uniformly and independently distributed across the monitored field. When practical application scenarios are considered, random deployment is a feasible and practical method, and sometimes it is the only feasible strategy [6]. But there may be a situation arises with random deployment, where, nodes may overlap on each other, so we may not guarantee full network coverage, and also, due to its randomness, it requires more number of sensor nodes to deploy a network as shown in below Figure .8

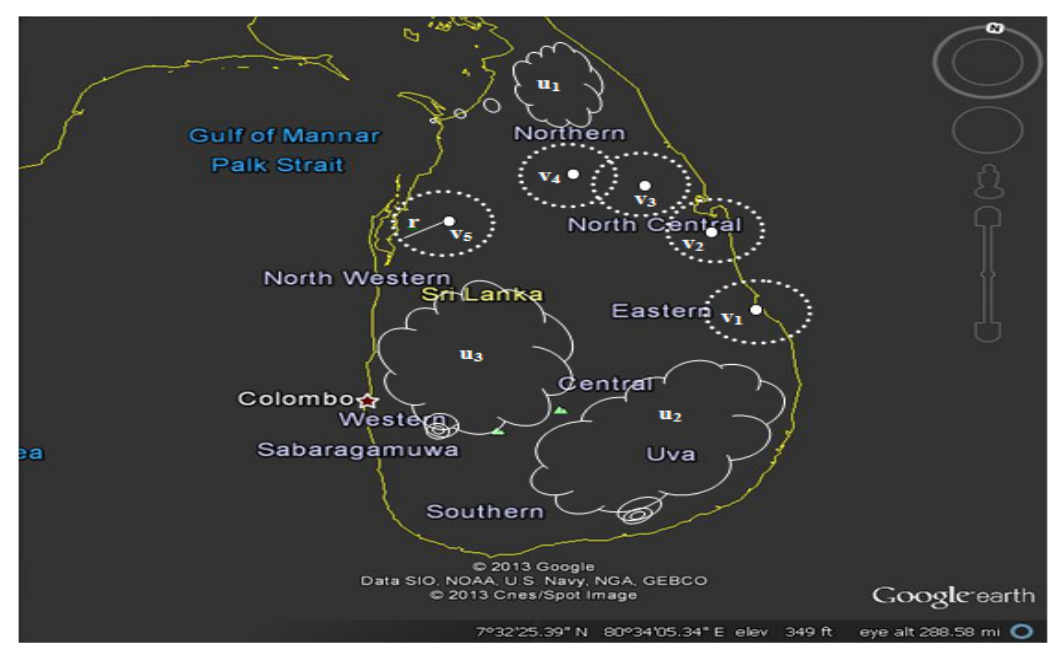

Figure 8. Expected target area coverage for a random deployment inside a real geographical map of Sri Lanka.

The above figure depicts the random deployment over target area $T_{r}$, where $u_{1}, u_{2}, u_{3}$ are the uncovered regions. Due to randomness and nodes overlapping, this deployment may not provide maximum network coverage.

\subsubsection{Analysis of Area Coverage for Grid Deployment}

Instead of deploying the nodes in a non-deterministic model (i.e. random deployment), now, we focus on the case, in which we partition the deployment region into various grids and analyse the maximum area coverage for various grid-based deployment schemes.

\section{Analysis of Area Coverage for Square Grid Deployment}

We are interested to calculate the maximum area coverage of sensor network while deploying the sensor nodes in square grid fashion within the target region $\boldsymbol{T}_{\boldsymbol{r}}$ of area, 64,740 $\mathrm{km}^{2}$, as shown in Figure. 9 .From this figure, if we consider a maximum possible unit square with edge points $\boldsymbol{c}_{\mathbf{1}}, \boldsymbol{c}_{\mathbf{2}}, \boldsymbol{c}_{\mathbf{3}}$ and $\boldsymbol{c}_{\mathbf{4}}$ of equal side length, then, from equation.

$$
\text { Area of Square }=\text { Side } * \text { Side }
$$

since, Side $=167.12 \mathrm{~km}$. Therefore, Area of a Square inscribed in a map $=$ 27929. 09

Thus, Area Coverage using square grid deployment $=27929.09 \mathrm{~km}^{2}$ 
International Journal of Ad hoc, Sensor \& Ubiquitous Computing (IJASUC) Vol.5, No.2, April 2014

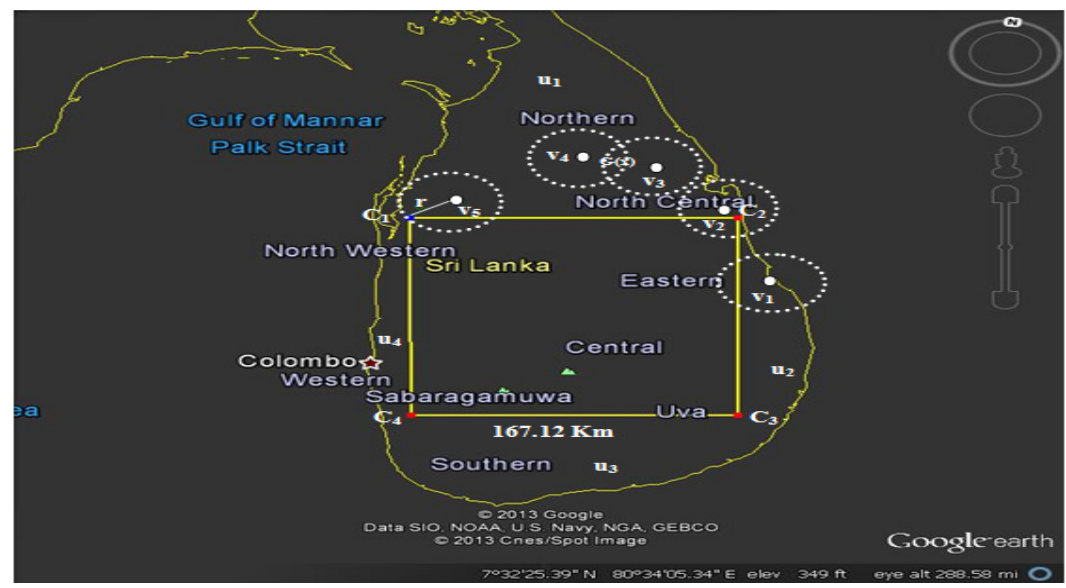

Figure 9. Expected target area coverage for a grid deployment in a unit square with side length 167.12 Km inside a real geographical map of Sri Lanka.

\section{Analysis of Area Coverage for Rectangular Grid Deployment}

If we deploy the sensor nodes in rectangular grid fashion within the target region $\boldsymbol{T}_{\boldsymbol{r}}$ of area, 64, $740 \mathbf{~ k m}^{2}$, as shown in below Figure.10, then the maximum area coverage can be obtained as, from equation (2), we know.

$$
\text { Area of Rectangle }=\text { width } * \text { height }
$$

From the below figure, we can observe that, the maximum possible rectangle with edge points $\mathbf{c}_{\mathbf{1}}, \mathbf{c}_{\mathbf{2}}, \mathbf{c}_{\mathbf{3}}$ and $\mathrm{c}_{\mathbf{4}}$ has, Width $=135.31 \mathrm{~km}$, Height $=261.41 \mathrm{~km}$

Area of a Rectangle inscribed in a map $=35371.38 \mathrm{~km}^{2}$

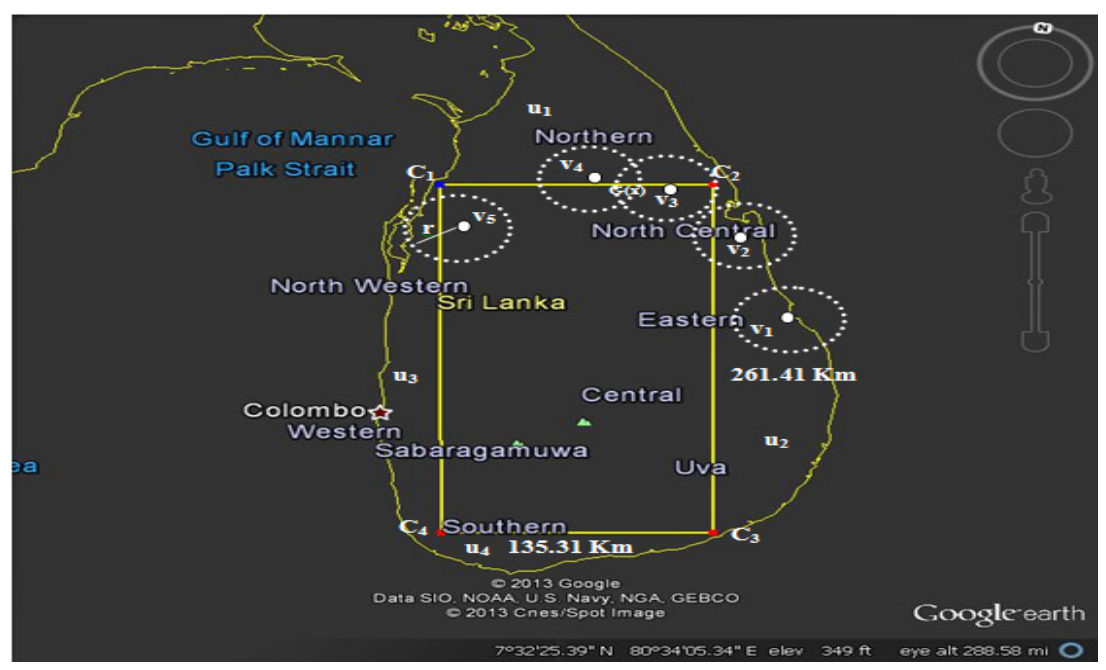

Figure 10. Expected target area coverage for a grid deployment in a rectangle with $135.31 \mathrm{Km}$, and $261.41 \mathrm{Km}$ lengths of each side respectively, inside a real geographical map of Sri Lanka. 
International Journal of Ad hoc, Sensor \& Ubiquitous Computing (IJASUC) Vol.5, No.2, April 2014

\section{Analysis of Area Coverage for Triangular Grid Deployment}

Now, if we consider the triangular shape inside the target region as shown in Figure.11, from equation (3), and we can calculate the maximum area covered by the best triangle inscribed in a target region. From the below figure, we can clearly notice that, the triangle consists of the edge point $\mathbf{c}_{1}, \mathbf{c}_{2}$ and $\mathbf{c}_{3}$ where,

$\mathrm{a}=\mathbf{c}_{\mathbf{1}}-\mathbf{c}_{\mathbf{2}}$ distance $=329.89 \mathrm{~km}$

$\mathrm{b}=\mathbf{c}_{\mathbf{1}}-\mathbf{c}_{\mathbf{3}}$ distance $=386.26 \mathrm{~km}$

$\mathrm{c}=\mathbf{c}_{2}-\mathbf{c}_{\mathbf{3}}$ distance $=209.58 \mathrm{~km}$

and,

Therefore,

$$
\mathrm{P}=\frac{329.89+386.26+209.58}{2}=462.87
$$

$$
A=\sqrt{462.87(462.87-329.89)(462.87-386.26)(462.87-209.58)}
$$

Hence,

Area of a Triangle inscribed in a map $=34540.27 \mathrm{~km}^{2}$

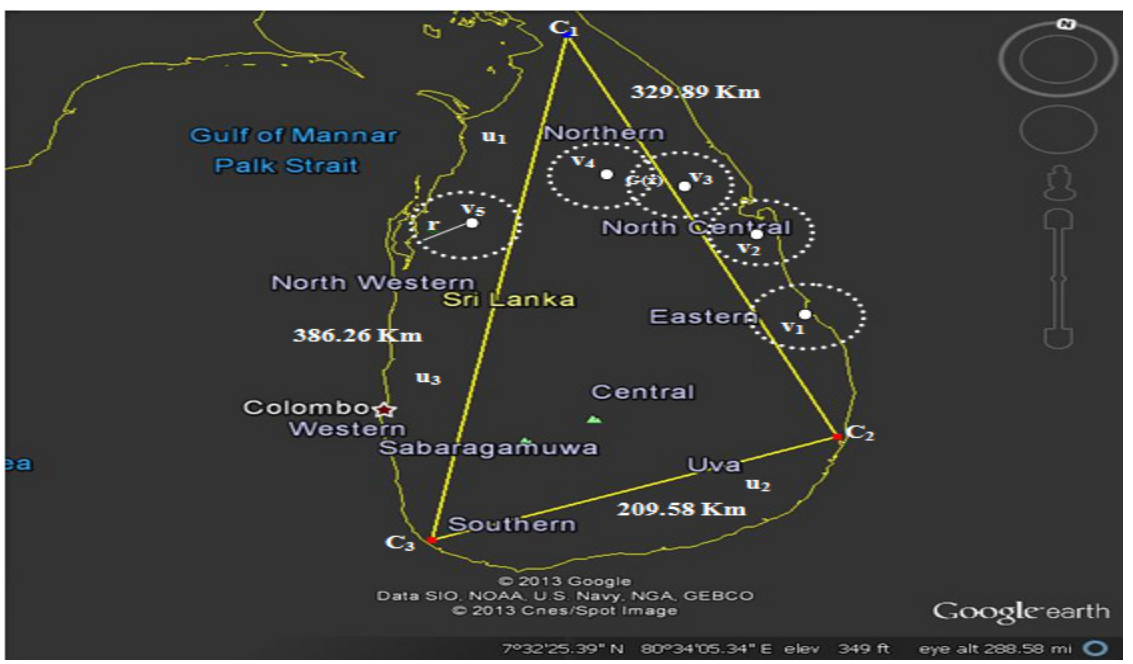

Figure 11. Expected target area coverage for a grid deployment in a triangle with $329.89 \mathrm{Km}$, $386.26 \mathrm{Km}$, and $209.58 \mathrm{~km}$ length of each side respectively, inside a real geographical map of Sri Lanka.

\section{Analysis of Area Coverage for Hexagonal Grid Deployment}

Now, we study the case, when we consider a hexagonal geometric shape inside the target region as shown in Figure.12. From equation (4), we know,

Where $\mathrm{S}$ in the length of any side

$$
\text { Area of a Hexagonal }=\frac{\mathrm{S}^{2} \mathrm{~N}}{4 \tan \left(\frac{180}{\mathrm{~N}}\right)}
$$

$\mathrm{N}$ is the number of sides

$\tan$ is the tangent function calculated in degrees

If we consider a maximum possible equilateral hexagon from the below figure with edge points, $\mathbf{c}_{1}, \mathbf{c}_{2}, \mathbf{c}_{3}, \mathbf{c}_{\mathbf{4}}, \mathbf{c}_{\mathbf{5}}, \mathbf{c}_{\mathbf{6}}$ and $\mathrm{s}$ ide length,$S=167.12 \mathrm{~km}$

Then, we can calculate,

Area of a Hexagonal inscribed in map $=32013.78 \mathrm{~km}^{2}$ 
International Journal of Ad hoc, Sensor \& Ubiquitous Computing (IJASUC) Vol.5, No.2, April 2014

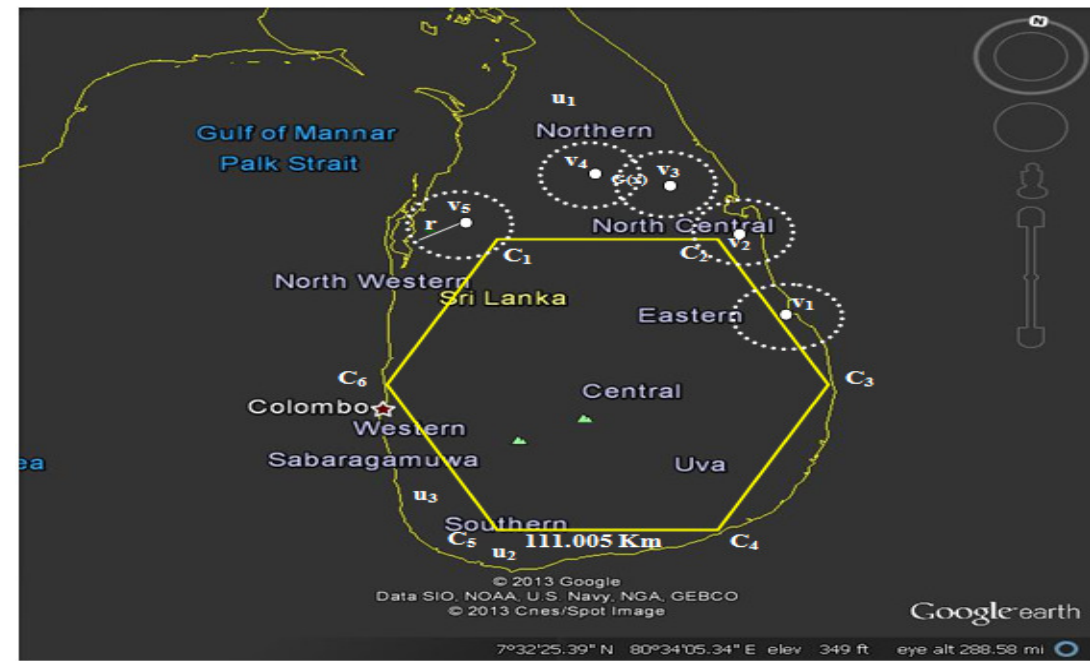

Figure 12. Expected target area coverage for a grid deployment in a unit hexagon with side length 167.12 Km inside a real geographical map of Sri Lanka

\subsection{Analysis of Area Coverage for Hybrid Deployment}

Now, we study the case, when we consider all of the above studied geometric shapes together inside the target region as shown in Figure.13.

Then, from equation (5), we know,

$$
\text { Hybrid Deployment }=\text { Area }(\mathrm{S} 1+\mathrm{S} 2+\mathrm{S} 3+\mathrm{S} 4+\mathrm{S} 5+\mathrm{S} 6+\mathrm{S} 7)
$$

Where $S_{1}, S_{2}, S_{3}, S_{4}, \ldots \ldots \ldots \ldots S_{7}$ are different geometric shapes inside the target region. From the below figure, we can notice, various geometric shapes, such as, triangle, square, hexagon, rectangle etc., where grid deployment can be possible. We can also observe various points, such as $u_{1}, u_{2}, u_{3}$ and $u_{4}$ are the uncovered region, where random deployment can be performed to cover the target region fully. Thus, we can calculate the area coverage for hybrid deployment as,

$$
\begin{aligned}
& \text { Area of } S_{1}=14879.35 \\
& \text { Area of } S_{2}=364.36 \\
& \text { Area of } S_{3}=23731.63 \mathrm{~km} \\
& \text { Area of } S_{4}=6920.56 \mathrm{~km} \\
& \text { Area of } S_{5}=12023.88 \mathrm{~km} \\
& \text { Area of } S_{6}=3439.05 \mathrm{~km} \\
& \text { Area of } S_{7}=2301.12 \mathrm{~km}
\end{aligned}
$$

Hybrid Deployment inscribed in a map $=63659.95 \mathrm{~km}^{2}$

Therefore area $=63659.95 \mathrm{Km} 2$ and uncovered region $u_{1}+u_{2}+u_{3}=1080.05 \mathrm{~km}^{2}$ 
International Journal of Ad hoc, Sensor \& Ubiquitous Computing (IJASUC) Vol.5, No.2, April 2014

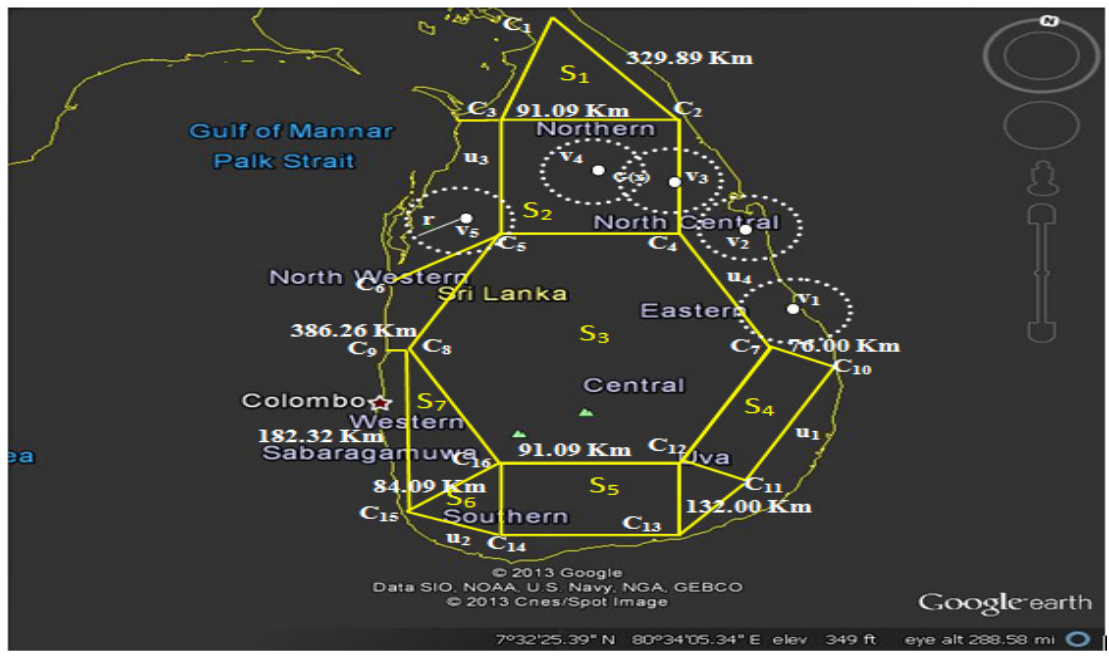

Figure 13. Expected target area coverage for a hybrid deployment inside a real geographical map of Sri Lanka.

\subsection{Comparative Analysis}

In order to deploy a sensor network with higher performance in terms of coverage, we have to gain the knowledge of various sensor node deployment schemes. As we discussed in the previous sections, the hybrid deployment exhibits higher network coverage than the remaining schemes. To make this point more clear, we assumed the area the wireless sensor network is to be deployed be a irregular shape real geographical map of Sri Lanka cuntry . We also analyzed the maximum area coverage for various deployment schemes by assuming basic geometric shapes inscribed in a irregular shape target region of size $64,740 \mathrm{~km}^{2}$, and depicted the simulation results in Figure.14.

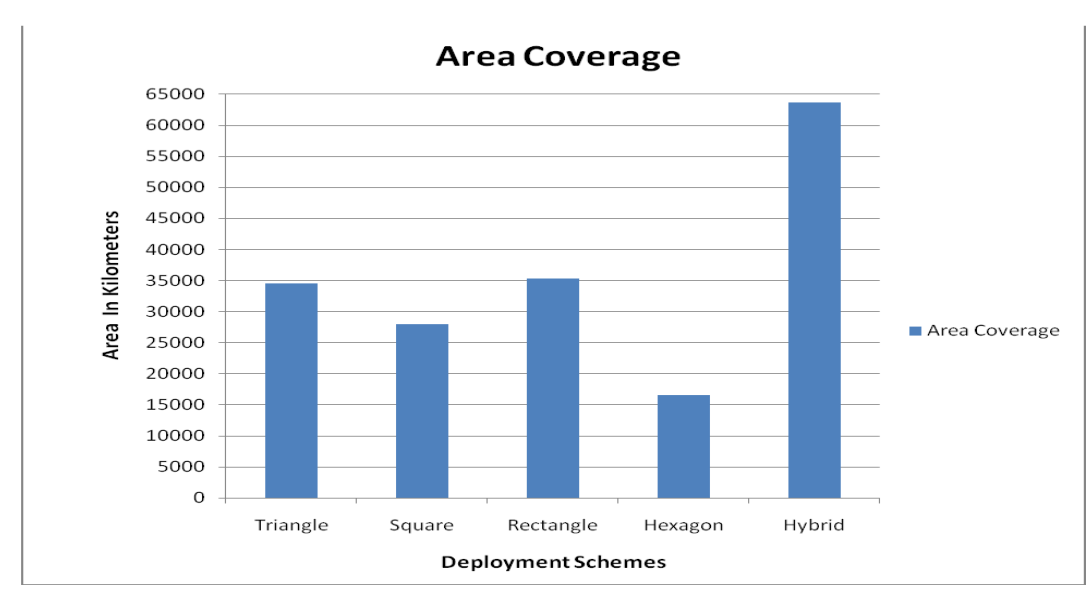

Figure 14. Simulation of network area coverage for various grid deployment schemes in WSNs

Here we calculated the maximum area coverage for each deployment scheme such as, squaregrid, rectangular-grid, triangular-grid and hexagonal-grid, and hybrid deployments. From the above simulation results, it is shown that, among all deployment schemes, hybrid deployment exhibits greater network coverage. 
International Journal of Ad hoc, Sensor \& Ubiquitous Computing (IJASUC) Vol.5, No.2, April 2014

\section{Conclusions}

In this paper we focused on deployment knowledge to improve the network coverage. We studied various node deployment schemes for WSNs. Under some deployment assumptions, we showed that the hybrid deployment provides better area coverage than other schemes.

\section{ACKNOWLEDGEMENTS}

The authors would like to thank to College of Engineering, Andhra University, Visakhapatnam and Sri Sai Aditya Institute of Science and Technology, Surampalem authorities for their constant support and cooperation!

\section{REFERENCES}

[1] Das, A.K.: Improving Identity-based Random Key Establishment Scheme for Large-scale Hierarchical Wireless Sensor Networks International Journal of Network Security, 13(3), 181-201 ( 2011).

[2] WANG, DU, and LIU 2009. ShortPK: A Short- Term Public Key Scheme for Broadcast Authentication in Sensor Networks. ACM Transactions on Sensor Networks, Vol. 6, No. 1, Article 9

[3] Crossbow Technology Inc., Wireless Sensor Networks, 2010. (http://www.xbow.com)

[4] Monica and Ajay K Sharma. Comparative Study of Energy Consumption for Wireless Networks based on Random and Grid Deployment Strategies. International Journal of Computer Applications (0975 - 8887), volume 6-no.1, September 2010.

[5] Coverage-Guaranteed Sensor Node Deployment Strategies for Wireless Sensor Networks Gaojuan Fan *, Ruchuan Wang, Haiping Huang, Lijuan Sun and Chao ShaJournal of Computer Applications (0975 - 8887), volume 6-no.1, September 2010.

[6] G Sanjiv Rao and V Vallikumari "The Fourth International Conference on Networks \& Communications (NETCOM-2012)"http://link.springer.com/chapter/10.1007\%2F978-1-4614-61548_35\#page-1

[7] Chun-Hsien Wu Yeh-Ching Chung .A Tiling-Based Approach for Directional Sensor Network Deployment.IEEE SENSORS 2010 Conference.

[8] B. Grunbaum and G. C. Shephard, "Tilings by Regular Polygons," Mathematics Magazine, vol. 50, no. 5 , pp. $227-247$

[9] Zhen Yu, and Yong Guan,A Key Management Scheme Using Deployment Knowledge for Wireless Sensor Networks. IEEE TRANSACTIONS ON PARALLEL AND DISTRIBUTED SYSTEMS, VOL 19, NO. 10, OCTOBER 2008.

[10] Liu, D., Ning, P., and Du, W. 2008. Group-based key predistribution for wireless sensor networks. ACM Trans. Sens. Netw. 4, 2, Article 11 (March 2008)

[11] The Network Simulator, NS-2, http://www.isi.edu/nsnam/ns/

[12] Akyildiz, I.F., Su, W., Sankarasubramaniam, Y.: A Survey on Sensor Networks IEEECommunications Magazine (August 2002)

[13] http://www.mannasim.dcc.ufmg.br/

[14] Sanji Rao, G., Valli Kumari, V.: A study on Deployment Schemes for Wireless Sensor Networks. In: AUC 3.0, The Third International Workshop on Ad Hoc \& Ubiquitous Computing, January 2-4. LNICST. Springer (2012)

[15] Lopez, Alonso, Sala, Marino, Haro: Simulation Tools for wireless sensor Networks

[16] www.google.com/earth/download-earth.html 
International Journal of Ad hoc, Sensor \& Ubiquitous Computing (IJASUC) Vol.5, No.2, April 2014

\section{AUTHORS}

G.Sanjiv Rao is currently working as Associate Professor in CSE Department, Sri Sai Aditya Institute of Science \& Technology Surampalem, AP, India. He is working towards Ph.D. at College of Engineering, Andhra University. He received is M.Tech from the same institute. His research interests are in the areas of Coverage over Wireless Sensor Networks.

Dr. V .Valli kumari is currently Professor in Department of Computer and Systems Engineering, College of Engineering, Andhra University Visakhapatnam, AP,India . She received her Ph.D. from Dept of CS\&SE, College of Engineering Andhra University. Her research interests include Security and Privacy issues in Data Engineering, Network Security and E-Commerce. She is a member of IEEE and ACM and fellow of IETE.

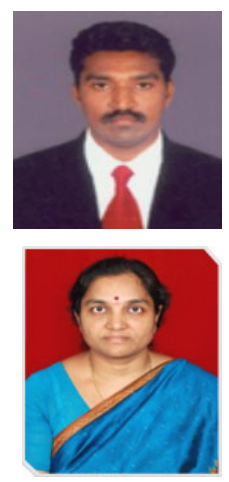

